\title{
Correlation between photoluminescence and positron annihilation spectra in porous silicon
}

\author{
C.C. Haung ${ }^{\mathrm{a}}$, I.M. Chang ${ }^{\mathrm{a}}$, Y.F. Chen ${ }^{\mathrm{a}, *}$, P.K. Tseng ${ }^{\mathrm{b}}$ \\ a Department of Physics, National Taiwan University, Taipei, Taiwan, ROC \\ ${ }^{\mathrm{b}}$ Department of Physics, Tamkang University, Taiwan, ROC
}

Accepted 16 August 1997

\begin{abstract}
We have performed two-dimensional angular correlation of electron-positron annihilation radiation (2D-ACAR) measurements on a series of porous silicon with photoluminescence (PL) peak energy in the range from 1.6 to $2.0 \mathrm{eV}$. The electron-positron momentum spectra of porous silicons can be well resolved into two peaks with different line width. The result shows two distinct momentum spectra for the studied samples. The samples with PL peak energy below $1.8 \mathrm{eV}$ exhibit a strong positronium signal in the momentum spectra, and the obtained result shows a good correlation with PL spectra as predicted by quantum confinement. However, the samples with PL peak energy above $1.8 \mathrm{eV}$ show little amount of positronium signal and the width of positronium peak remains almost constant. This behavior can be explained by the formation of silicon compounds on the surface of pores. With the result of these measurements, we suggest that the PL mechanism is different for the porous silicon with PL peak below and above $1.8 \mathrm{eV}$. For the PL peak below $1.8 \mathrm{eV}$, the emission arises from silicon nano-crystal, so that the PL spectrum highly depends on the nano-crystal size. However, for the PL above $1.8 \mathrm{eV}$, we suggest that the luminescence is dominated by the silicon compounds on the surface of the pores which block the free volume for positronium annihilation. Our suggested model can be used to resolve several difficulties in the existing PL spectra. (C) 1998 Elsevier Science B.V. All rights reserved.
\end{abstract}

PACS: $78.70 . \mathrm{Bj} ; 61.72 . \mathrm{Qq} ; 61.66 \mathrm{Bi}$

Keywords: Porous silicon; Positron; Photoluminescence

\section{Introduction}

Porous silicon obtained by the partial electrochemical dissolution in HF solutions was first discovered by Uhlir in 1956 [1]. Strong PL can be

\footnotetext{
* Corresponding author. Fax: 88623639984 ; e-mail: yfchen@phys.ntu.edu.tw.
}

observed in the visible range at room temperature under ultraviolet and blue-green wavelength laser excitation [2]. This suggests the possibility of its application in new generation Si-based optoelectronic devices. To explain the effective PL behavior, various reports have been devoted to investigate its origin. Several reports suggested that the porous silicon has wire-like structure and confines the carriers in one-dimensional nano-crystal wires or 
zero-dimensional particles $[2,3]$. However, not all of the experimental results agree with the quantum-confinement model. Thus, several authors pointed out that the chemical reactants remained in the porous structure dominate the PL mechanism [4-6]. Each model has its advantage in some specific cases, but hardly to explain all experimental results. To date the PL mechanism of porous silicon is still not conclusively accepted.

To ascertain the PL mechanism of porous silicon, firstly, it is necessary to understand the microstructure of the porous layer. To observe the morphology of porous silicon, several experimental techniques have been used, such as high-resolution transmission electron microscopy (TEM) and scanning tunneling microscopy (STM) $[7,8]$. However, these techniques are confined to the near surface region. Because the porous silicon can be easily oxidized, we believe that in the sample preparing process or even exposed in the air there exists an oxidized layer on the surface of porous layer. This makes the observation of the porous structure by the techniques mentioned above very difficult.

New possibilities are opened for 2D-ACAR technique which provides the two-dimensional electron-positron momentum density with high resolution. The positron with the positive charge repelled by the ion cores shows a very good sensitivity to the defects or voids in condensed matter [9]. This technique, long established as a major tool for studying atomic defects and voids, has emerged as a potentially powerful tool for providing information about the voids and defects. The formation of positronium has been proved to exist in porous silicon by several authors $[10,11]$. From the uncertainty principle, the full-width at half-maximum (FWHM) $\Delta P$ of the positronium momentum distribution will have an inversed relation to the space size $\Delta X$ occupied by the positronium, which can provide the information of the pore size distribution. Thus, by measuring the annihilation of the trapped positronium we can obtain the morphology of porous silicon.

To date only a few positron studies have been performed on porous silicon. Cruz et al. first found that the intermediate positron lifetime in porous silicon strongly depends on sample preparation
[12]. Itoh et al. found a long lifetime component ( $25 \mathrm{~ns}$ ) and a sharp Doppler broadening spectrum, which prove the formation of positronium in porous silicon [11]. Suzuki et al. found that the longlived positron lifetime component in porous silicon changes with the sample and annealing temperatures [13]. In our previous report, we first measured the momentum spectrum in porous silicon and proved that the narrow component in the momentum spectrum belongs to the trapping of positronium in the free volume of pores [10].

Recently, we obtained a rather interesting result from the measurement of the positron lifetime and 2D-ACAR spectrum on a series of lightly doped porous silicon samples which were prepared with solutions of different HF concentrations [14]. Both the positron lifetime and 2D-ACAR experiments show that the pore size decreases and pore number increases with decreasing HF concentration. The result clearly demonstrates that the less concentrated HF solution not only enlarges the porosity but also increases the number of the smaller protrusions which are hardly observed by STM and TEM measurements.

In this paper, we simultaneously perform 2DACAR and PL measurements on a series of porous silicon samples. We are able to discover the correlation between the microstructure information obtained from 2D-ACAR spectra and the recombination processes in PL spectra. A possible PL mechanism in porous silicon is suggested, and it can be used to resolve several difficulties in the existing PL data.

\section{Experimental method}

The samples in our measurements were etched by using p-type $\left(\begin{array}{lll}1 & 0 & 0\end{array}\right)$ oriented single-crystal silicon wafers with resistivity $10-20 \Omega \mathrm{cm}$. Aluminum was evaporated onto the back as an electric contact. To obtain the different PL spectra shown in Fig. 1, samples were prepared under different conditions. Table 1 shows the preparing parameters and the PL peak energies.

The electron positron momentum distributions of the porous silicon were carried out by a 2D-ACAR system. The system consists of two 


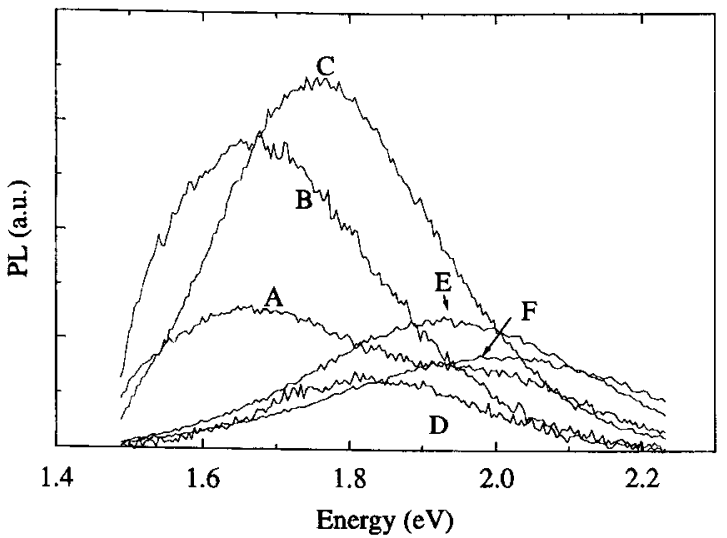

Fig. 1. The PL spectra of the porous silicon samples prepared under different condition.

position-sensitive Anger-camera gamma-ray detectors separated from the sample by $17.11 \mathrm{~m}$ and $18.6 \mathrm{~m}$, respectively. Each Anger camera consists of a $48 \mathrm{~cm}$-diameter and $1 \mathrm{~cm}$-thick $\mathrm{NaI}(\mathrm{Tl})$ scintillator with 64 photomultiplier tubes optically coupled to the scintillator. 2D-ACAR spectra were recorded as the angular distribution of the two gamma ray with $0.6 \mathrm{mrad}$ angular resolution. Each spectrum was collected by about $10^{7}$ events in $3 \mathrm{~d}$ under $10^{-6}$ Torr.

\section{Results and discussion}

Fig. 2a shows a typical spectrum of the twodimensional electron-positron momentum distribution for porous silicon with PL peak below $1.8 \mathrm{eV}$. Comparing with the 2D-ACAR spectrum of bulk silicon as shown in Fig. 2b, we can see that an extra sharp distribution is found in the center of the spectrum of porous silicon. The contribution of the sharp peak is about $2-20 \%$ of the total signal
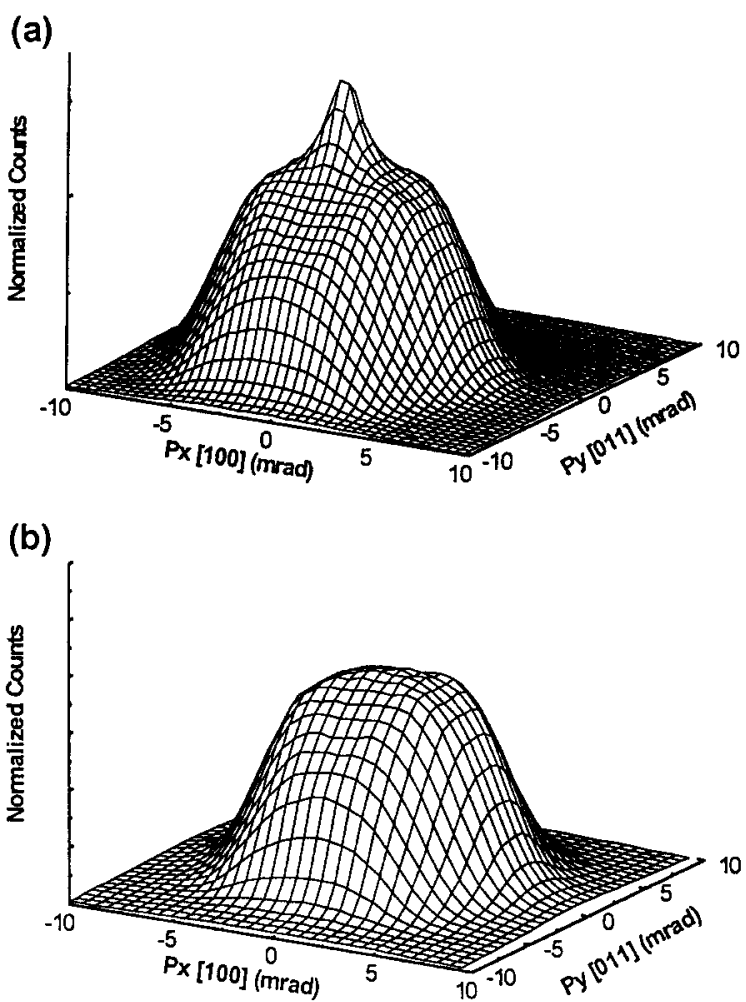

Fig. 2. Two-dimensional electron-positron momentum distributions of (a) porous silicon and (b) silicon wafer.

which depends on the samples. The measurement of the silicon wafer after etching out the porous silicon layer has also been performed and the result is the same as that of the original silicon wafer. We, therefore, conclude that the sharp peak is due to the existence of the porous layer. Because the extra sharp signal shows no explicit anisotropic behavior due to the random distribution of the pores, all the two-dimensional data $N(\operatorname{Pr}, \theta)$ is averaged through $\theta$ into $N(\operatorname{Pr})$ to reduce the statistical error. The $N(\mathrm{Pr})$ spectrum can be fitted by a bulk-silicon

Table 1

The PL peak energies and the etching condition of the investigated porous silicon samples

\begin{tabular}{lllllll}
\hline & A & B & C & D & E & F \\
$\mathrm{HF}: \mathrm{H}_{2} \mathrm{O}: \mathrm{C}_{2} \mathrm{H}_{6} \mathrm{O}$ & $2: 3: 5$ & $1: 1: 1$ & $3: 3: 4$ & $1: 1: 2$ & $6: 9: 20$ & $6: 9: 25$ \\
PL peak energy(eV) & 1.66 & 1.67 & 1.76 & 1.84 & 1.93 & 2.0 \\
\hline
\end{tabular}

Note: Etching current $20 \mathrm{~mA}$, etching time $30 \mathrm{~min}$. 


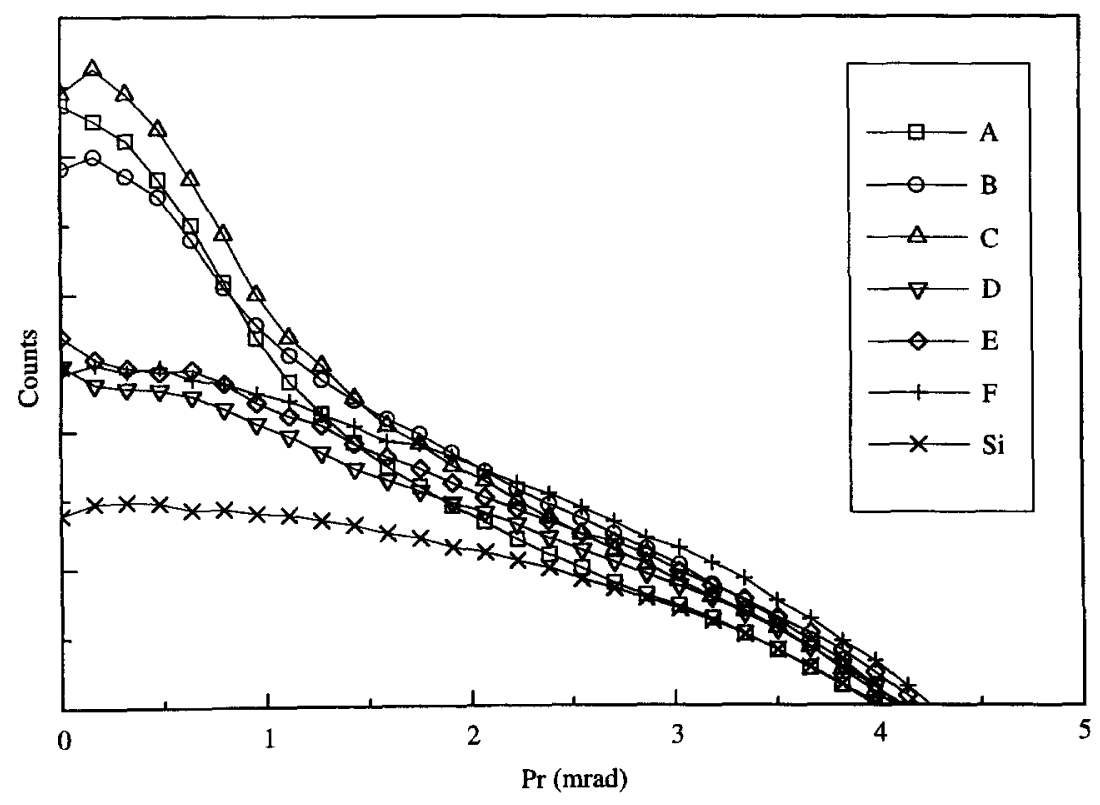

Fig. 3. Electron-positron momentum spectra of the bulk silicon wafer and porous silicon prepared under different condition.

spectrum and two Gaussian distributions with a narrow and a wider peak as shown in our previous report [10].

Fig. 3 shows the measured momentum spectra of our studied samples and the window is set to the central peaks to emphasize the difference between the samples. It is obvious that there are two kinds of momentum spectra. From 0 to $1 \mathrm{mrad}$ the samples A-C clearly have a stronger signal than the samples D-F. It is interesting that the PL peak energies of the samples $\mathrm{A}-\mathrm{C}$ are all below $1.8 \mathrm{eV}$, and that of the samples $\mathrm{D}$ and $\mathrm{E}$ are above $1.8 \mathrm{eV}$. These results suggest that the porous silicons with PL peak below and above $1.8 \mathrm{eV}$ have quite different microstructure which results in the two distinct momentum spectra observed. In order to discuss the effect of microstructure on the optical properties, the relations between the FWHM and intensity and PL peak energy are shown in Figs. 4 and 5 for the narrow and the wider peak, respectively. From Fig. 4 we can see that for PL peak energy smaller than $1.8 \mathrm{eV}$, the FWHM of the narrow peak increases with PL peak energy, and no apparent change occurs after the PL peak exceeds $1.8 \mathrm{eV}$. The intensity of the narrow peak increases with $\mathrm{PL}$ peak energy below $1.8 \mathrm{eV}$ and decreases with $\mathrm{PL}$
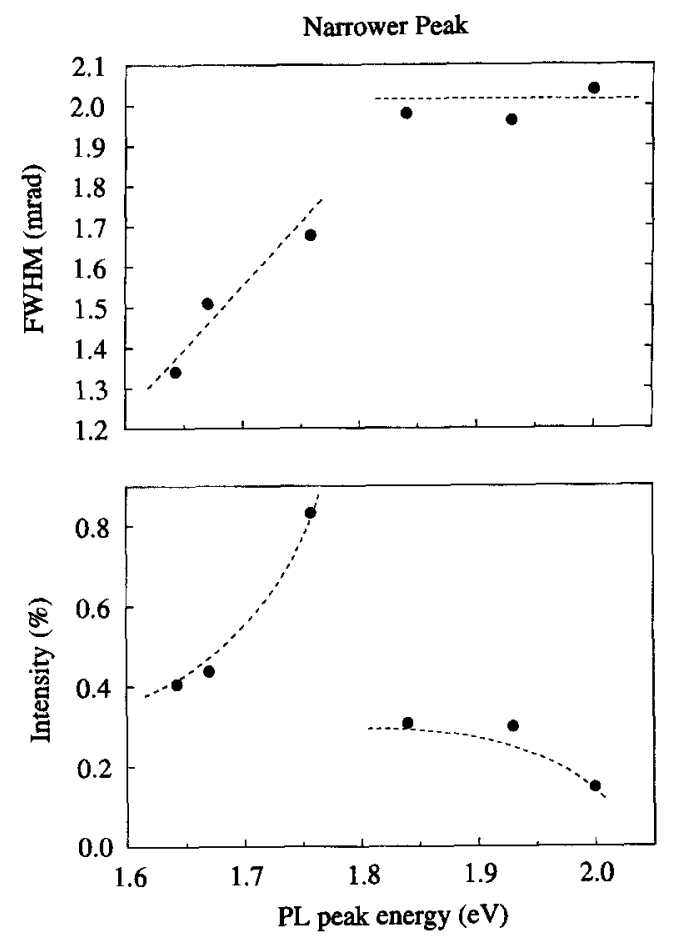

Fig. 4. The correlation between the PL peak energy and the FWHM and intensity of the narrow peak in the momentum spectra of porous silicon. Dashed lines are used to guide eye only. 

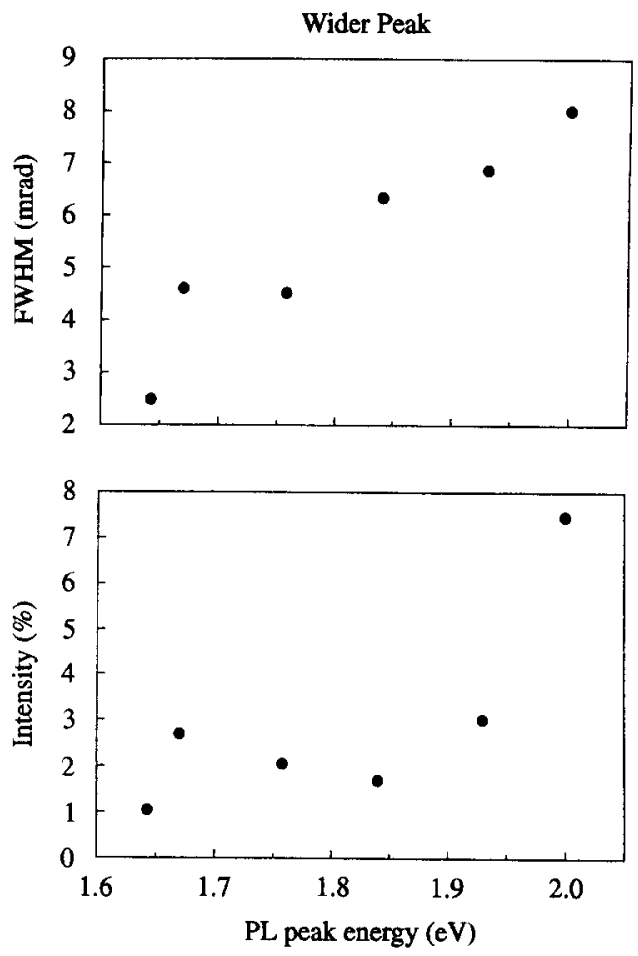

Fig. 5. The correlation between the PL peak energy and the FWHM and intensity of the wider peak in the momentum spectra of porous silicon.

peak energy above $1.8 \mathrm{eV}$. From Fig. 4. we find that the FWHM of the wider peak increases with PL peak energy and the intensity begins to increase above $1.8 \mathrm{eV}$. In our previous report [10] we have proved that the narrow peak in the porous silicon momentum spectrum belongs to the trapping of positronium inside the free volume of pores. The increase of FWHM and intensity of the momentum spectrum of the narrow peak means that the average size of pores decreases and the number of pores increases. To explain this result, we adopt the formation mechanism of porous layer proposed by Beale et al. [15]. The growth of the porous structure does not only enlarge the pores size but also produces new branches of pores. Since the growth of the porous structure is dominated by producing new branches, the number of pores will increase and the average size will decrease due to the large amount of new branches. This is reflected in the increasing of the FWHM and intensity of the momentum spectrum of the narrow peak. In addition, the formation of new branches of pores will divide the silicon crystal into smaller parts. So the increasing of new branches will decrease the size of nanocrystal silicon. From the quantum confinement model, the PL is determined by the nano-crystal size of silicon and the smaller crystal size corresponds to a more blue PL spectrum. So the porous silicon with more blue PL spectrum has a stronger and wider positronium peak in the electron positron momentum spectrum. Our results suggest that the blue shift of PL peak energy below $1.8 \mathrm{eV}$ is dominated by the creation of structures of smaller size in the silicon crystal in connection with the appearance of new protrusions.

The trend of the FWHM and intensity of positronium is different between the samples with $\mathrm{PL}$ peak above and below $1.8 \mathrm{eV}$. The FWHM of the samples with PL peak above $1.8 \mathrm{eV}$ has no explicit change and the intensity decreases with the increasing of PL peak energy. The model proposed above fails to explain the result of the samples with PL peak above $1.8 \mathrm{eV}$. From the decreasing of the intensity of the positronium peak in the momentum spectra, we suggest that high concentration of silicon compounds forms in the pores. The existence of silicon compounds has been proposed in many previous reports [4-6]. These silicon compounds will block the pores and provide the defect sites for the trapping of the positron. Thus, the silicon compounds in the pores will reduce the formation probability of positronium and increase the probability of positron trapping in the defect space. The samples with more silicon compounds therefore have a smaller signal of the narrow peak. Because the trend of the intensity of the wider peak is opposite to that of the narrow peak, we suggest that the wider peak is probably due to the positron trapping in the defect states. However, the origin of the wider peak still needs further investigation.

The results of 2D-ACAR study in porous silicon show that for the PL peak energy below $1.8 \mathrm{eV}$, the blue shift of the PL spectra can be attributed to the reduction of the nanocrystal size. This suggests quantum confinement. However, for the samples with PL peak larger than $1.8 \mathrm{eV}$ we found that the charge trapping is dominated by the surface chemistry of the pores. This finding can provide a very important clue for the understanding of the $\mathrm{PL}$ 
mechanism. Previously, it has been shown that for the samples with PL peak above $1.8 \mathrm{eV}$, the peak energy does not depend on the size of the silicon crystallite [16], which rules out the possibility of the quantum confinement model. It has been shown that the PL spectra strongly depends on the surface properties. For instance, cycle shifts in the PL spectra can be obtained when one changes the solution [17]. Thus, the result of our 2D-ACAR study is consistent with the fact that for the samples with PL peak above $1.8 \mathrm{eV}$, the charge trapping and annihilation are dominated by the surface compounds. We, therefore, suggest that the PL in porous silicon arises from two different processes. For the samples with PL peak below $1.8 \mathrm{eV}$, the emission can be described by the effect of quantum confinement. For the samples with PL peak above $1.8 \mathrm{eV}$, the PL is determined by the surface species. It is interesting to note that the existing PL data in a recent review article can be explained by our model [18]. We believe that a continuous study based on our suggestion may lead to fruitful information for the understanding of PL mechanism in porous silicon.

\section{Conclusions}

In conclusion, we have investigated the momentum spectra by 2D-ACAR measurements on a series of porous silicon sample with different PL spectrum. Two extra peaks with different line width are found in these spectra. The narrower peak is attributed to positronium trapping inside the free volume of pores and the wider peak is suggested to be the result of positron trapping on the surface silicon compounds. Two distinct momentum spectra have been found for the samples with different PL spectrum. The samples with PL peak below $1.8 \mathrm{eV}$ have sharper positronium signal in the electron-positron momentum distribution. The FWHM and intensity of the positronium peak increase with the PL peak energy, which indicates the decrease in the average pores size and the increase in the number of the pores. This result shows that the amount of smaller new protrusions increases and divides the silicon into many smaller sizes which makes the blue shift in the PL spectra. For the samples with
PL peak above $1.8 \mathrm{eV}$, the intensity of positronium decreases with increasing PL peak energy. This is explained by the increasing of silicon compounds formed on the surface of pores. From these results, we suggest that the PL mechanism below $1.8 \mathrm{eV}$ can be described by quantum confinement effect and the mechanism above $1.8 \mathrm{eV}$ is dominated by the recombination through silicon compound at the pore surface. In addition, we have proposed a new avenue for the simultaneous study of positron and optical spectra which can help in understanding of the physical properties of porous silicon and should also be applicable to the study materials.

This work is partially supported by the National Science Council of the Republic of China.

\section{References}

[1] A. Uhlir, Bell Syst. Tech. J. 35 (1956) 333.

[2] L.T. Canham, Appl. Phys. Lett. 57 (10) (1990) 1046.

[3] A. Nishida, K. Nakagawa, H. Kakibayashi, Jpn. J. Appl. Phys. 31 (1992) L1219.

[4] M.S. Brandt, H.D. Fuchs, M. Stutzmann, H. Weber, M. Cardona, Solid State Commun. 81 (1992) 307.

[5] S.M. Prokes, O.J. Glembocki, V.M. Bermudez, R. Kaplan, L.E. Friedersdorf, P.C. Searson, Phys. Rev. B 45 (1992) 13788.

[6] D.J. Wolford, B.A. Scott, J.A. Reimeer, J.A. Bradley, Physica 117B\&118B (1983) 9220.

[7] R.L. Smith, S.D. Collins, J. Appl. Phys. 71 (1992) R1.

[8] V.P. Parkhutik, J.M. Albella, J.M. Martinez-Duart, J.M. Gomez-Rodriguez, A.M. Baro, V.I. Shershulsky, Appl. Phys. Lett. 62 (1993) 366.

[9] S.J. Wang, Y.C. Jean, in: D.M. Schrader, Y.C. Jean (Eds.), Positron and Positronium Chemistry, Elservier, Amsterdam, 1988, p. 255.

[10] C.C. Haung, I.M. Chang, Y.F. Chen, P.K. Tseng, Physica B 228 (1996) 374.

[11] Y. Itoh, H. Murakami, A. Kinoshita, Appl. Phys. Lett. 63 (1993) 2798.

[12] R.M. De la Cruz, R. Pareja, Phys. Status Solidi A 111 (1989) 463.

[13] R. Suzuki, T. Mikado, H. Ohgaki, M. Chiwaki, T. Yamazaki, Phys. Rev. B 49 (1994) 17484.

[14] C.C. Haung, I.M. Chang, Y.F. Chen, P.K. Tseng, unpublished.

[15] M.I.J. Beale, J.D. Benjamin, M.J. Uren, N.G. Ghew, A.G. Cullis, J. Crystal Growth 73 (1970) 622.

[16] D.J. Lockwood, A. Wang, B. Bryskiewicz, Solid State Commun. 89 (1994) 587.

[17] K. Li, C. Tsai, J. Sarathy, J.C. Campbell, Appl. Phys. Lett 62 (1993) 3192

[18] Yoshihiko Kanemitsu, Phys. Rep. 263 (1995) 1. 